\title{
Besteht ein Zusammenhang von Alkoholkonsum und sexuellem Risikoverhalten? Eine Diskussion konzeptioneller Aspekte am Beispiel HIV-infizierter Männer mit homosexuellem Verhalten
}

\author{
Niklowitz, Matthias ; Eich-Höchli, Dominique
}

\begin{abstract}
Besteht ein Zusammenhang zwischen Alkoholkonsum und sexuellen Risikokontakten? Führt Alkohol zu Risikokontakten? Es lassen sich zahlreiche Studien finden, die diese Frage bejaht haben. Ebenso lassen sich Studien finden, die mit modifizierten Forschungskonzepten diese Befunde nicht bestätigen konnten. Unter Verwendung von ereignisunspezifischen und situationsspezifischen Konzepten wurden diese Fragen bei einer Stichprobe von 64 HIV-infizierten Männern mit homosexuellem Verhalten untersucht. Einen signifikanten Zusammenhang von Alkoholkonsum und sexuellem Risikoverhalten konnten wir mit dem ereignisun-spezifischen Ansatz nicht feststellen. Auch Personen, die sexuelle Kontakte unter Alkoholeinfluss eingegangen waren (situationsspezifisches Konzept), gingen nicht überdurchschnittlich häufiger Risikokontakte ein. Die Personen hingegen, die unter Alkoholkonsum riskante sexuelle Praktiken eingegangen waren, erreichen sowohl hinsichtlich des Alkoholkonsums als auch des allgemein erfassten Schutz- und Risikoverhaltens lediglich Durchschnittswerte. Erst konkrete Zusatzfragen machen es möglich, sie als Personen mit Risikoverhalten zu identifizieren. Für Untersuchungen zum sexuellen Schutz- und Risikoverhalten erscheint die Kombination sich ergänzender Forschungskonzepte zweckmäs$\operatorname{sig}$
\end{abstract}

DOI: https://doi.org/10.1007/bf01592325

Posted at the Zurich Open Repository and Archive, University of Zurich

ZORA URL: https://doi.org/10.5167/uzh-156083

Journal Article

Published Version

Originally published at:

Niklowitz, Matthias; Eich-Höchli, Dominique (1997). Besteht ein Zusammenhang von Alkoholkonsum und sexuellem Risikoverhalten? Eine Diskussion konzeptioneller Aspekte am Beispiel HIV-infizierter Männer mit homosexuellem Verhalten. Sozial- und Präventivmedizin, 42(5):286-297.

DOI: https://doi.org/10.1007/bf01592325 
Matthias Niklowitz, Dominique Eich-Höchli

Departement innere Medizin, Abteilung für Infektionskrankheiten und Spitalhygiene, Universitätsspital Zürich

\section{Besteht ein Zusammenhang von Alkohol- konsum und sexuellem Risikoverhalten? Eine Diskussion konzeptioneller Aspekte am Beispiel HIV-infizierter Männer mit homosexuellem Verhalten}

\section{Zusammenfassung}

Besteht ein Zusammenhang zwischen Alkoholkonsum und sexuellen Risikokontakten? Führt Alkohol zu Risikokontakten? Es lassen sich zahlreiche Studien finden, die diese Frage bejaht haben, Ebenso lassen sich Studien finden, die mit modifizierten Forschungskonzepten diese Befunde nicht bestätigen konnten. Unter Verwendung von ereignisunspezifischen und situationsspezifischen Konzepten wurden diese Fragen bei einer Stichprobe von 64 HIV-infizierten Măniern mit homosexuellem Verhalten untersucht Einen signifikanten Zusammenhang von Alkoholkonsum und sexuellem Risikoverhalten konnten wir mit dem ereignisunspezifischen Ansatz nicht feststellen. Auch Personen, die sexuelle Kontakte unter Alkoholeinfluss eingegangen waren (situationsspezifisches Konzept), gingen nicht überdurchschnittlich häufiger Risikokontakte ein. Die Personen hingegen, die unter Alkoholkonsum riskante sexuelle Praktiken eingegangen waren, erreichen sowohl hinsichtlich des Alkoholkonsums als auch des allgemein erfassten Schutz- und Risikoverhaltens lediglich Durchschnittswerte. Erst konkrete Zusatzfragen machen es möglich, sie als Personen mit Risikoverhalten zu identifizieren. Für Untersuchungen zum sexuellen Schutz- und Risikoverhalten erscheint die Kombination sich ergänzender Forschungskonzepte zweckmässig.

Solange kurative Heilungsmöglichkeiten gegen Aids fehlen, kommt der Identifizierung von Einflüssen und Situationen, die für die Weitergabe des Virus förderlich sind, grosse Bedeutung zu. Ein wichtiger Infektionsweg sind sexuelle Kontakte. Ungeschützte anale Kontakte, die Anzahl der Sexualpartner, der HIV-Serostatus des Partners sowie Nichtgebrauch von Kondomen korrelieren hoch mit der HIV-
Serokonversion ${ }^{1}$. Schon sehr früh war die psychosoziale Aids-Forschung daran interessiert, die Umstände, die zu sexuellen Risikokontakten führen, zu untersuchen, denn nicht immer fruchten die Präventionsbemühungen: Bei britischen Männern mit homosexuellem Verhalten, die 1993 bis 1995 an Gay Pride-Festivals teilnahmen, konnte keine Änderung des sexuellen Risikoverhaltens festgestellt werden $^{2}$. Die Frage nach Einflussgrössen, die zu sexuellen Risikokontakten führen, ist also nach wie vor aktuell.

Als eine dieser Grössen gilt Alkoholkonsum. Das Wissen um einen Zusammenhang von Alkoholkonsum und riskantem Sexualverhalten ist weit verbreitet. Zentral ist das Konzept der „Enthemmung“, es impliziert, dass der Gebrauch von Alkohol (und bestimmten anderen Drogen) zu erhöhter Inkaufnahme von Risiken und zu weniger Vorsicht führt ${ }^{3}$. Vielzitierter Ausgangspunkt für die empirische Untermauerung dieses $\mathrm{Zu}$ sammenhangtheorems ist die Arbeit von Stall und Kollegen ${ }^{4}$. Bei weiteren Untersuchungen ${ }^{5-19}$ mit gleichem Ergebnis bei unterschiedlichen Studienpopulationen wurde dieser Befund bestätigt. Shafer und Boyer beispielsweise resümieren: "The best predictor of sexual risk behavior was alcohol and drug use" 9 .

Es lassen sich aber auch eine Reihe von Arbeiten mit gegenteiligen Ergebnissen finden. Untersuchungen, in der die situative Verbindung von Alkoholkonsum und unsafe Sex entweder in tagebuchähnlichen Aufzeichnungen ${ }^{20}$ oder anhand je eines exemplarischen Kontaktes bei jungen Männern homosexueller Orientierung, 
der einmal safe, einmal unsafe erfolgt war, verneinen Zusammenhänge ${ }^{21}$. Bestätigt wurde dieser Befund von Temple ${ }^{22}$, der gezielt den Stellenwert des Alkoholkonsums im situativen Kontext des letzten Sexualkontaktes erfasst hatte. Klienten von Prostituierten neigen nicht $\mathrm{zu}$ riskanterem Sexualverhalten unter Alkoholund/oder Drogeneinfluss ${ }^{23,24}$. Die mit Männern mit homosexueller Orientierung durchgeführten Untersuchungen von Bolton et al. ${ }^{25}$ und Weatherburn ${ }^{26}$ zeigen keinerlei Verbindung von Alkohol und Risikoverhalten. Leigh und Miller ${ }^{27}$ stellten fest, dass Personen, die sexuelle Aktivitäten und Alkoholkonsum kombiniert hatten, keine erhöhte Wahrscheinlichkeit von Risikokontakten aufwiesen. Eine mit Daten der Schweizerischen HIV-Präventionsstudie durchgeführte Untersuchung ${ }^{28}$ konnte keinen Zusammenhang zwischen Alkoholtrinkmenge und Risikokonstanten mit GelegenheitspartnerInnen feststellen. Möglicherweise besteht aber ein indirekter Zusammenhang: McCusker et al. ${ }^{29}$ stellten einen Rückgang von riskanten Sexualpraktiken bei Personen fest, die auch ihren Alkoholbzw. Marihuanakonsum reduziert hatten. Traeen and Lewin ${ }^{30}$ nennen den Lebensstil, der mit erhöhtem Alkohol- und Tabakkonsum verbunden ist, als Risikofaktor.

Die WHO schliesst in ihrem Positionspapier zu Alkohol und HIV/Aids ${ }^{3}$ den widersprüchlichen Forschungsstand mit der Feststellung, dass bestimmte Personen offensichtlich mehr Risiken eingehen als andere. Diese Personen, die ein erhöhtes Risikoverhalten aufweisen, trinken auch mehr und öfter. Ebenso wurde aber auch gezeigt, dass der Gebrauch oder Nichtgebrauch von Alkohol das individuelle Risikoverhalten in bestimmten Situationen nicht beeinflusst. Zu einer klaren Aussage über den Zusammenhang von sexuellem Risikoverhalten und
Alkoholkonsum gelangt der WHO-Bericht nicht.

Hingegen bestehen Hinweise, wonach der Wahl des Konzeptes und der Operationalisierung des sexuellen Risikoverhaltens offenbar entscheidend Bedeutung zukommt: In ihrer Kritik der Arbeit von Stall et al. ${ }^{5}$ stellte Leigh $^{31}$ bei ihrer Studienpopulation fest, dass alleine ein Wechsel der Definition des Risikokontaktes das Ergebnis veränderte.

Die Befunde lassen somit die Annahme begründet erscheinen, dass Alkoholkonsum und Risikoverhalten zwar zumindest statistisch zusammenhängen, Alkoholkonsum aber nicht zu erhöhtem sexuellem Risikoverhalten führt. Die Hypothesen formulieren wir wie folgt:

Erstens: Alkoholkonsum und sexuelles Risikoverhalten korrelieren positiv.

Zweitens: Personen, die sexuelle Kontakte unter Alkoholeinfluss angeben, gehen nicht häufiger Risikokontakte ein als Personen, die keine solchen Kontakte aufweisen. Bevor wir zur Methodik gelangen, vergleichen wir die bisher verwendeten Untersuchungskonzepte.

\section{Untersuchungskonzepte}

Für die Untersuchung des Zusammenhangs von Alkohol und Risiko lassen sich drei Konzepte einführen ${ }^{32}$ : Erstens gibt es das ereignisunspezifische Konzept. Bei diesem werden allgemeine Masse über Sexualverhalten (Anzahl Kontakte, teilweise differenziert nach Partnertyp), sexuelles Risikoverhalten, Alkohol- und Drogenkonsum und u.U. weitere Einflussgrössen erhoben und statistisch miteinander in Beziehung gesetzt. Beispiele sind die Studien von Penkower et al. ${ }^{10}$ oder Plant et al. ${ }^{24}$. Zweitens das ereignisspezifische Konzept, bei dem von den Studienpersonen die Umstände der einzelnen sexuellen Kontakte festgehalten werden. Die bereits oben refe- rierten Studien von Leigh ${ }^{20}$ oder Temple und Kollegen ${ }^{22}$ bilden Beispiele für diese Konzeption. Einer Zwischenform dieser Konzeptionen entsprechen drittens die situationsspezifischen Konzepte. Bei diesen werden, ähnlich der zweiten Konzeption, allgemeine Masse erfasst, sie werden aber ergänzt um gezielte Fragen, ob und wie oft bestimmte Einflüsse, hier: Alkoholkonsum und sexuelles Risikoverhalten, gleichzeitig erfolgten. Beispiele hierfür sind die bekannte Stall-Studie von $1986^{4}$ sowie die von Bagnall und Kollegen ${ }^{6}$.

Alle drei Konzepte weisen Schwachpunkte auf ${ }^{32-34}$ : Das ereignisunspezifische Konzept lässt offen, ob überhaupt ein kausaler Zusammenhang zwischen Alkoholkonsum und Risikokontakt besteht. Ein gleichzeitiges Vorkommen von Alkoholkonsum und unsafe Sex liesse sich auch anders interpretieren, etwa als Ausdruck von Suche nach Nervenkitzel. Beim ereignisspezifischen Konzept sind mögliche Schwierigkeiten bei der Erhebung zuverlässiger Daten aus der Vergangenheit zu nennen. Das Führen von Tagebüchern durch die Studienpersonen bietet eine Abhilfe (beispielsweise ${ }^{20,26}$ ). Das Vorhandensein konfundierender Variablen lässt sich durch diese Erhebungsmethode nicht ausschliessen, aber dieser Einwand gilt auch für die beiden anderen Designs. Beim dritten Konzept bleibt offen, inwieweit Zusammenhänge zwischen Alkoholkonsum und Risikokontakten einen Artefakt darstellen, da mit zunehmender Anzahl sexueller Kontakte ganz einfach auch die Möglichkeit steigt, dass diese in Verbindung mit Alkoholkonsum erfolgen.

Wir gehen in dieser Untersuchung zunächst vom allgemeinsten, dem ereignisunspezifischen Konzept, aus und korrelieren für die Prüfung der ersten Hypothese verschiedene Risikoindikatoren mit unterschiedlichen Massen zum Alkoholkonsum. Dann prüfen wir die zweite 
Hypothese unter Berücksichtigung des situationsspezifischen Konzeptes und untersuchen, ob Personen, die sexuelle Kontakte unter Alkoholeinfluss genannt hatten, eher Risikokontakte eingegangen waren als die anderen Studienpersonen. Hier stellt sich zudem die Frage, ob allgemeine Indikatoren zum Risikoverhalten sowie Alkoholkonsummasse diese Personen erfassen, m.a.W., ob sie einen höheren Alkoholkonsum oder einen höheren Wert bei den Risikoindikatoren aufweisen. Schliesslich erkunden wir die unter Alkoholeinfluss unterlassenen Schutzmassnahmen und untersuchen diese einzelnen Fälle genauer. Auch hier steht die Frage im Vordergrund, wie präzise sich die situationsspezifischen Angaben zum Risikoverhalten mit den ereignisunspezifischen Indikatoren decken.

\section{Methode}

In den Jahren 1989 und 1990 wurden von den 742 registrierten, asymptomatischen HIV-positiven Personen der Zürcher Kohorte der Schweizerischen HIV-Kohortenstudie 137 Personen, die sowohl bei der Halbjahreskontrolle erschienen als auch den Einschlusskriterien entsprachen, an der Abteilung Infektionskrankheiten und Spitalhygiene des Universitätsspitals Zürich bezüglich einer freiwilligen Teilnahme an dieser prospektiven Untersuchung angefragt. 20 Personen mussten ausgeschlossen werden: 17 hielten trotz dreimaliger Vereinbarung Termine nicht ein, drei weitere Personen beendeten das Interview vorzeitig.

Einschlusskriterien waren schriftliche Einwilligung, Teilnahme an der Schweizerischen HIV-Kohortenstudie, Teilprogramm $\mathrm{A}^{35}$, Alter über 16 Jahre, asymptomatisches Infektionsstadium, ausreichende Deutschkenntnisse, kein aktueller Drogenkonsum oder Gefängnisaufenthalt.
Von den verbleibenden 117 Studienpersonen gehören $64(55 \%)$ der Betroffenengruppe der Männer mit homosexuellem Verhalten an. Nur diese Personen werden in die vorliegende Untersuchung eingeschlossen.

\section{Instrumente}

Die hier verwendete Instrumente wurden von der Schweizerischen HIV-Kohortenstudie Teilprogramm B ${ }^{35,36}$ übernommen:

- HIPF-Klinikfragebogen, enthält Angaben zu sexuellem Schutz- und Risikoverhalten. Getrennt werden hetero- und homosexuelle Kontakte erhoben, weiter unterteilt nach Praktiken (oral, vaginal, anal) und Partnertypen (feste Partner, Gelegenheitspartner, Prostituierte, Freier/Stricher). Von jedem dieser Typen wird - je nach sexueller Praktik - die Anzahl der Kontakte in den letzten sechs Monaten vor der Befragung erhoben, ergänzt um die Frage, ob diese immer mit Präservativ oder ungeschützt mit Ejakulation erfolgt sind (s. Anhang).

- HIPS-Fragebogen zum Selberausfüllen, enthält Fragen zu psychosozialen Faktoren, Alkoholund Drogenkonsum in Verbindung mit sexuellen Kontakten. In diesem Fragebogen sind die Fragen zu sexuellen Kontakten unter Alkohol- und Drogeneinfluss integriert. Es wird erfasst, ob und wie oft die Studienpersonen in den sechs Monaten vor der Befragung sexuelle Kontakte unter Alkoholoder Drogenkonsum eingegangen waren und wie gross der prozentuale Anteil aller Kontakte unter dem Einfluss einer dieser Substanzen mit den verschiedenen oben genannten Partnertypen geschätzt wurde. Ein zweiter Abschnitt erfasst anschliessend, ob die Studienpersonen unter Alkohol- bzw. Drogeneinfluss Vorsichtsmassnahmen bezüglich "safer sex" unterlassen hatten, die sie nüchtern eingehalten hätten. Es wird abschlies- send eruiert, welche Praktiken, die nüchtern vermieden worden wären, mit welchem Partnertyp eingegangen worden sind (s. Anhang).

Operationalisierung der Risikokontakte

Oft wird Risikoverhalten dichotomisiert. Eine Unterteilung in „sichere“ und „unsichere Kontakte" kann auf praktisch jedes Risikokonstrukt angewandt werden, ob es nun Kondomgebrauch, ungeschützte Kontakte, mehrere PartnerInnen oder insertiver Analverkehr (wie z.B. Robins und Kollegen für den Pittsburgher Teil der $\mathrm{MACS}^{37}$ ) ist. Auf der anderen Seite bestehen zahllose Risikoindikatoren siehe $^{2}$ oder in der Schweiz $\left.{ }^{38}\right)$.

Dazwischen stehen Indikatoren, die den Kondomgebrauch (oft) in drei bis fünf Stufen erfassen (beispielsweise aus der MACS ${ }^{39}$ sowie ${ }^{1,40,41}$ ). Wir verwenden hier drei ereignisunspezifische Indikatoren:

- Risikokontakte (dichotom), getrennt für feste und Gelegenheitspartner. Bei diesem Indikator überprüfen wir, ob bei Sexualpraktiken mit substantieller Infektionswahrscheinlichkeit (aktiver oder passiver Analverkehr)

1. immer Kondome gebraucht wurden bzw.

2. der Gebrauch mindestens einmal unterlassen worden ist.

Diesen Index bezeichnen wir als dichotomen Risikoindikator.

- Risikokontakte (vierstufig), ${ }^{39}$ :

1. keine analen Kontakte;

2. sämtliche anale Kontakte geschützt;

3. teils geschützte, teils ungeschützte anale Kontakte;

4. immer ungeschützte anale Kontakte. 
Auch bei diesem Indikator werden aktiver oder passiver Analverkehr berüicksichtigt. Die niedrigste Kategorie schliesst alle Personen ein, die keine oder lediglich orale Kontakte genannt hatten. Wir bezeichnen dieses Risikomass als vierstufigen Risikoindikator.

- Risikoindikator (vierstufig) der Chicagoer MACS-Forschergrup$\mathrm{pe}^{42}$.

1. Kein Risiko: Keine sexuellen Kontakte oder Partner.

2. Niedriges Risiko: Sexuelle Kontakte mit Partner(n), aber entweder kein rezeptiver Analverkehr oder rezeptiver Analverkehr nur mit festem Partner und konsistentem Kondomgebrauch.

3. Erhöhtes Risiko: Entweder konsistenter Kondomgebrauch bei rezeptivem Analverkehr mit mehreren Partnern oder ungeschützter rezeptiver Analverkehr, beschränkt auf den festen Partner.

4. Hohes Risiko: ungeschützter rezeptiver Analverkehr, mehrere Partner.

Für die folgenden Ausführungen gilt für diesen Index die Kurzbezeichnung MACS-Risikoindikator.

Operationalisierung des Alkoholkonsums

Wir berücksichtigen zur Erfassung ereignisunspezifischen Trinkverhaltens die folgenden allgemeinen Masse:

1. Trinkhäufigkeit: „Wie häufig trinken Sie alkoholische Getränke?"

2. Trinkmenge: „Wenn Sie Alkohol trinken, wieviel trinken sie dann pro Gelegenheit?"

3. Rausch: „In den letzten 6 Monaten, wie oft hatten Sie einen "Rausch"?"

4. MACS-Alkohol-Trinkfrequenz: Die Alkohol-Trinkfrequenz der MACS errechnet sich aus der
Anzahl Drinks (= Gläser mit alkoholischem Getränk) pro Monat, also aus dem Produkt von Trinkhäufigkeit und Trinkmenge. Dieses Rohmass wird trichotomisiert: niedriger Konsum (bis 12 Drinks pro Monat), mittlerer Konsum (13 bis 59 Drinks) und hoher Konsum (über 59 Drinks) ${ }^{42}$.

Sexuelles Risikoverhalten und Alkoholkonsum

Nach den ereignisunspezifischen allgemeinen Massen zu sexuellem Risikoverhalten und Alkoholkonsum ermöglichen die situationsspezifischen Angaben zu sexuellem Risikoverhalten unter Alkoholeinfluss eine Überprüfung der Vali- dität der Angaben. Die Fragen zu den konkreten Folgen des Alkoholkonsums gehen inhaltlich über den situationsspezifischen Ansatz hinaus, da konkret nach den unterlassenen Schutzmassnahmen und Praktiken gefragt wird. Als ereignisspezifisches Vorgehen können sie aber nicht aufgefasst werden, da weitere Begleitumstände (die beispielsweise in Tagebuchaufzeichnungen festgehalten werden können) im standardisierten Fragebogen nicht erfasst worden sind.

Mit der Frage „Hatten Sie während der letzten 6 Monate sexuelle Kontakte unter Alkoholeinfluss?" wurde situationsspezifisch der Zusammenhang vom sexuellem Risikoverhalten und Alkohol-

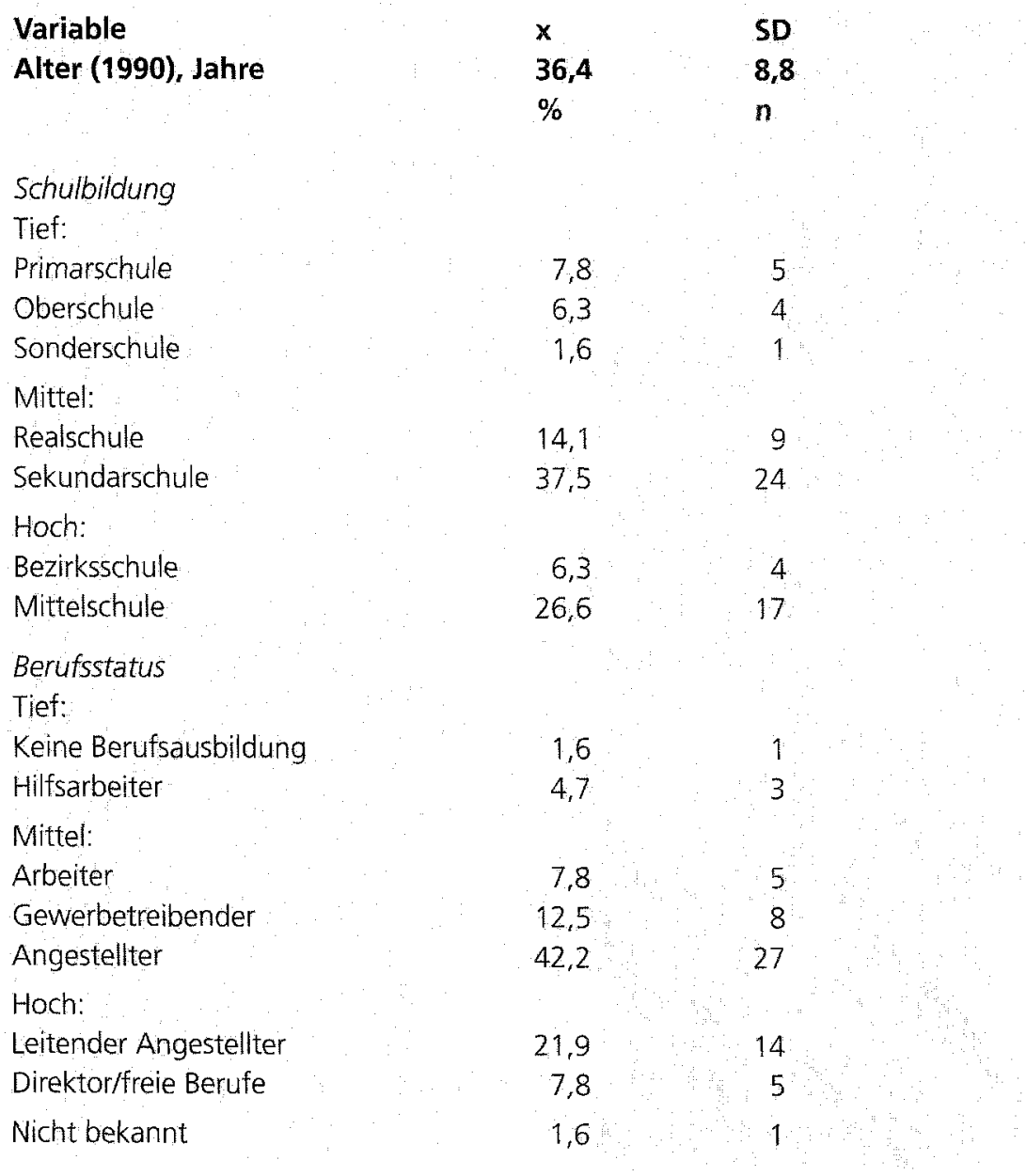

Tabelle 1. Soziodemografische Angaben $(n=64)$. 
konsum untersucht. Wenn diese Frage bejaht worden war, wurde erfragt,

- mit welchem Partnertyp (feste, Gelegenheits- und anonyme Partner)

- wieviele Kontakte (in fünf Antwortvorgaben abgestuft) unter Alkoholeinfluss stattgefunden hatten.

Ein zweiter Bereich von Fragen richtete sich auf die Folgen des Alkoholkonsums auf das sexuelle Risikoverhalten: Es wurde erfasst, ob die Studienpersonen durch den Alkoholeinfluss

- bestimmte Sexualpraktiken eingegangen waren bzw.

- Schutzmassnahmen (Präservativgebrauch) unterlassen hatten.

\section{Resultate}

Sexuelles Schutz- und Risikoverhalten

Von den 64 Studienpersonen hatten 61 in den letzten sechs Monaten vor der Befragung sexuelle Kontakte (oral, und/oder anal) mit festen oder Gelegenheitspartnern. Kontakt mit sich prostituierenden Personen oder Strichern wurden keine genannt. Die soziodemografischen Angaben der ausschliesslich männlichen Stichprobe finden sich in Tabelle 1, die Angaben zum sexuellen Schutz- und Risikoverhalten in Tabelle 2.

Alkoholkonsum der Stichprobe: Im Vergleich mit der seronegativen Stichprobe von Sieber ${ }^{38}$ sind Trinkhäufigkeit und -menge der hier befragten HIV-Infizierten praktisch identisch. Einzig bei der Anzahl der Räusche geben mehr HIV-Infizierte an, dass sie in den letzten sechs Monaten vor der Befragung nie einen gehabt hätten. Im Vergleich mit Erhebungen zum Alkoholkonsum in der Schweiz ${ }^{43}$ sind die Personen dieses Sample bei den Extremgruppen (gar kein bzw. täglicher Alkoholkonsum) unter-, bei der Gruppe mit mindestens einmaligem wöchentlichem Konsum übervertreten. Für den Grossteil unserer Studienpopulation gehört Alkoholkonsum zum Alltag.

Sexuelle Risikokontakte unter Alkoholeinfluss: Elf Personen des Sample hatten sexuelle Kontakte unter Alkoholeinfluss angegeben, vier von ihnen unterliessen unter Alkoholeinfluss Schutzmassnahmen. Von diesen vier Personen nannten zwei aktiven Analverkehr mit Präservativen und je eine aktiven bzw. passiven Analverkehr ohne Präservativ. In diesen beiden Fällen kam es unter Alkoholeinfluss zu sexuellen Kontakten mit substantieller Infektionswahrscheinlichkeit.

Vergleich der Indikatoren: Die drei Risikoindikatoren korrelieren signifikant untereinander: Der dichotome und vierstufige Indikator mit (Spearmans Rangkorrelations- koeffizient) $\mathrm{R}=0,75$ ( $\mathrm{p}<0,001)$, der dichotome mit dem MACSIndikator mit $\mathrm{R}=0,48(\mathrm{p}<0,001)$ und der MACS-Risikoindex mit dem vierstufigen Rissikomass mit $\mathrm{R}=0,31(\mathrm{p}<0,05)$. Eine Studienperson weist beim MACS-Index den Maximalwert auf (vgl. Tabelle 2). Auch von den anderen beiden Indikatoren wurde dieser Person die jeweils höchste Risikostufe zugeordnet. Sie trank ein- bis zweimal pro Woche ein bis zwei Gläser mit alkoholischen Getränken und liegt im Bereich der niedrigsten MACS-Alkoholkonsumstufe. Sexuelle Kontakte unter Alkoholeinfluss wurden keine genannt und demzufolge waren auch keine Vorsichtsmassnahmen infolge von Alkoholkonsum unterlassen worden.

\section{Hypothesenprüfung}

Bei den Zusammenhängen der drei Risikoindikatoren mit Alkohol-

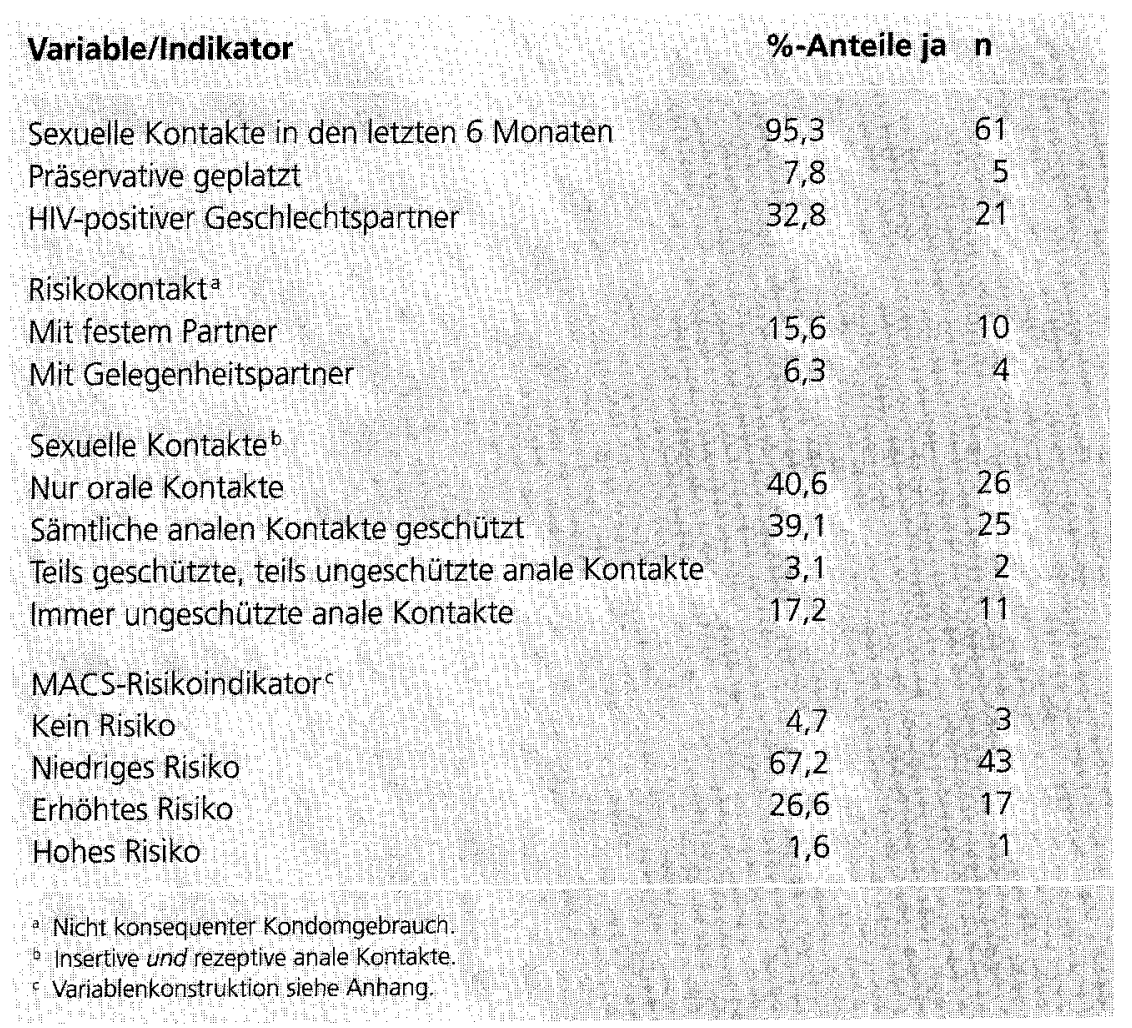

Tabelle 2. Sexuelles Schutz- und Risikoverhalten $(n=64)$. 


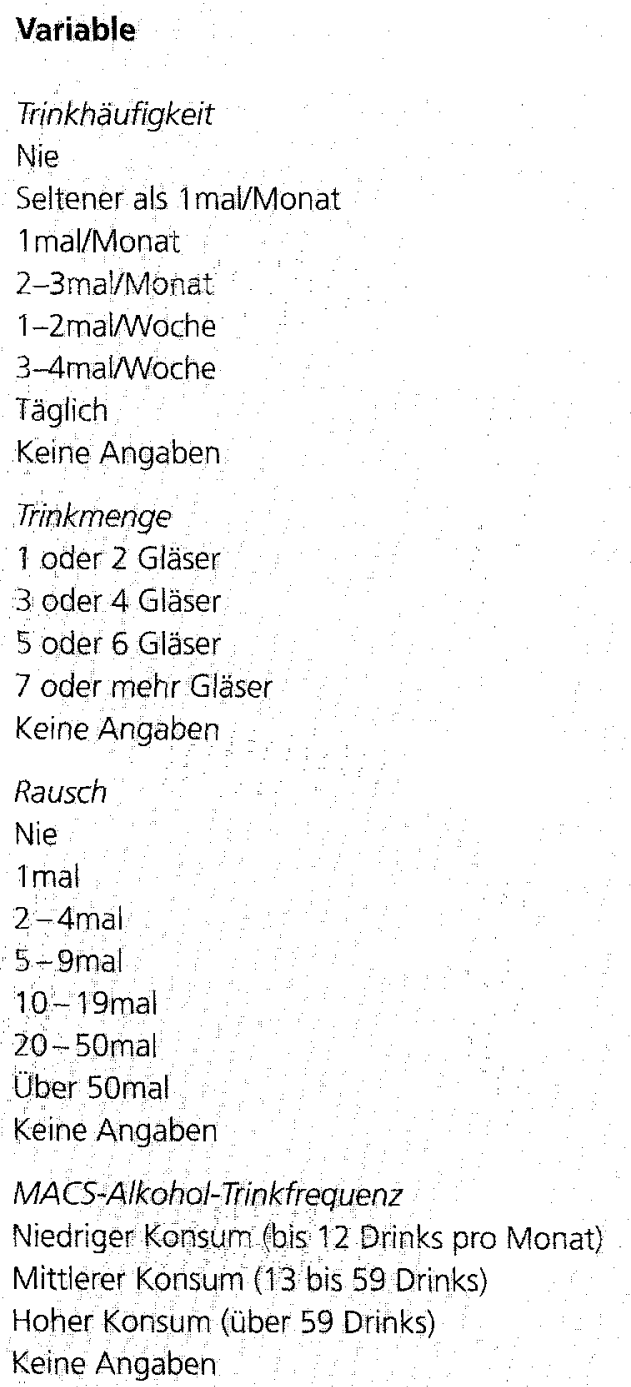

Tabelle 3. Alkoholkonsum $(n=64)$.

\section{Variable}

Sexuelle Kontakte unter Alkoholeinfluss

Insgesamt (alle Partnertypen)
Davon
Mit festem Partner
Mit Gelegenheitspartner

Unter Alkoholkonsum eingegangene Praktiken und unterlassene Schutzmassnahmen

Insgesamt

Praktiken:

Aktiver Analverkehr mit Präservativ

Aktiver Analverkehr ohne Präservativ

Passiver Analverkehr ohne Präservativ

$\begin{array}{rr}1,6 & 1 \\ 6,3 & 4 \\ 6,3 & 4 \\ 17,2 & 11 \\ 28,1 & 18 \\ 29,7 & 19 \\ 9,4 & 6 \\ 1,6 & 1\end{array}$

$\begin{array}{rr}48,4 & 31 \\ 39,1 & 25 \\ 7,8 & 5 \\ 1,6 & 1 \\ 3,1 & 2\end{array}$

40,6

23,4

17,2

4,7

6,3

3,1

1,6

3,1

43,8

46,9

7,8

1,6 . 1 8 9 6.

1.
1 1.

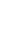




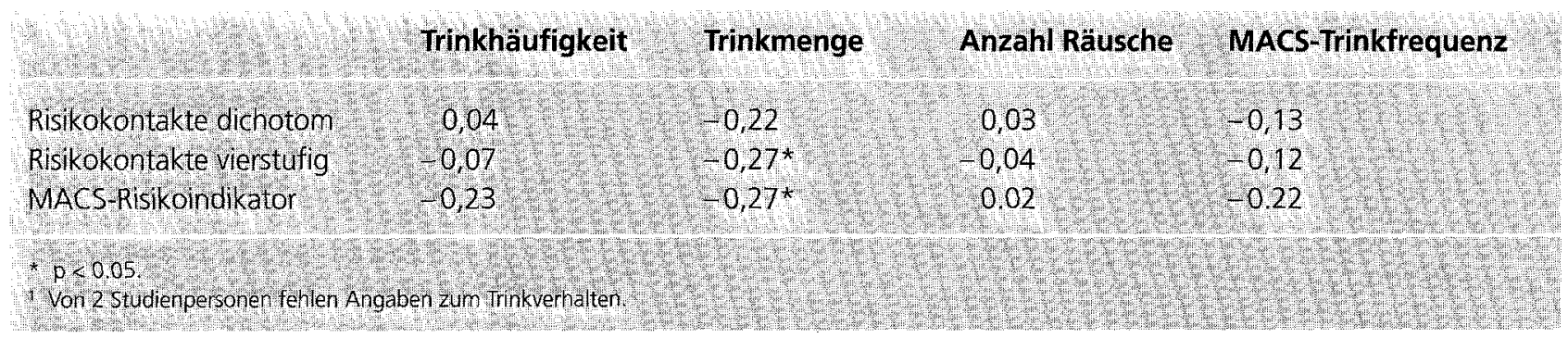

Tabelle 5. Sexuelle Risikoindikatoren und Alkoholkonsum, Spearman-Rangkorrelationen $(n=62)^{7}$.

war und den anderen Studienteilnehmern zeigen sich teilweise signifikante Unterschiede: Bei allen Trinkmassen lagen diese elf Personen durchschnittlich niedriger als die anderen Probanden: Sie nannten in den letzten sechs Monaten vor der Befragung signifikant weniger Räusche (U-Test: p < $0,001)$, sie tranken eine signifikant geringere Menge $(p<0,05)$. Beim MACS-Alkoholkonsummass wiesen sie ebenfalls - nicht signifikant $(p=0,06)$ - tiefere Werte auf. Personen mit sexuellen Kontakten unter Alkoholeinfluss trinken also weniger und nennen seltener Räusche als die anderen Studienteilnehmer.

\section{Unterlassene Schutzmassnahmen unter Alkoholeinfluss}

Von den vier Personen, die unter Alkoholkonsum Schutzmassnahmen unterlassen hatten, finden sich beim dichotomen sowie beim vierstufigen Indikator je zwei in der höchsten Risikokategorie. Beim MACS-Indikator erreicht eine Person die dritte Stufe "modified high risk", drei weitere die nächsttiefere "low risk"-Kategorie. Wir können feststellen, dass sich effektives Verhalten und Indikatorenwerte nicht immer entsprechen.

\section{Diskussion}

Besteht ein Zusammenhang zwischen Alkoholkonsum und sexuellen Risikokontakten? Führt Alko- hol zu Risikokontakten? Diese Fragen haben wir bei einer Stichprobe von 64 HIV-infizierten Männern mit homosexuellem Verhalten untersucht. Einen positiven Zusammenhang von sexuellem Risikoverhalten und Alkoholkonsum konnten wir nicht feststellen. Ebenso wies die Minderheit von Probanden, die sexuelle Kontakte unter Alkoholeinfluss eingegangen war, keinen erhöhten Anteil von Personen mit Risikokontakten auf.

Alkoholkonsum und sexuelles Risikoverhalten korrelieren bei dieser Stichprobe - teilweise signifikant - negativ miteinander: Bei Personen mit hohem Alkoholkonsum liegt der Anteil mit Risikokontakten tendenziell tiefer, bei solchen mit tiefem Konsum eher höher. Von einem gesicherten statistischen Zusammenhang können wir nicht sprechen. Gar kein $\mathrm{Zu}$ sammenhang besteht zwischen der Anzahl Räusche und dem Risikoverhalten. Alkohol wird nur dann verhaltenswirksam, wenn es zu einem Rausch kommt, somit die Handlungskontrolle vermindert ist und gleichzeitig ein sexueller Kontakt zustandekommt. Alkoholtrinkmenge und Trinkhäufigkeit hingegen geben auch Aufschluss über einen Lebensstil. Zudem kann eine Toleranzentwicklung auftreten oder der Betreffende gewöhnt sich an den Umgang mit psychoaktiven Substanzen und sein Alkoholkonsum beeinflusst nicht die spezifischen Verhaltensroutinen. Diese Interpretation wird durch unsere Untersuchung bei den Personen gestützt, die Alkoholkonsum und sexuelle Kontakte kombiniert hatten, wir konnten bei diesen kein erhöhtes Risikoverhalten feststellen. Wir gelangen damit zur Schlussfolgerung, dass Alkoholkonsum keinen Einfluss auf sexuelle Risikokontakte hat.

Diese Aussage stützt sich auf zwei sich ergänzende Untersuchungskonzepte. Die von uns verwendeten Fragebögen HIPF und HIPS lassen sowohl ereignisunspezifische als auch situationsspezifische Vorgehensweisen zu und ermöglichen zudem eine Validierung von Angaben, die sehr persönlich und nicht „objektiv“ überprüfbar sind. Es zeigte sich, dass die Personen mit sexuellen Kontakten unter Alkoholeinfluss weder durch überdurchschnittliche Werte bei den Risikoindikatoren noch durch übermässigen Alkoholkonsum auffallen. Von diesen Personen gingen nur zwei ungeschützte Kontakte ein, bei denen eine reelle Infektionswahrscheinlichkeit bestand. Damit stellt sich die Frage, mit welcher Konzeption diese Personen am besten erfasst werden. Das ereignisunspezifische greift hier offenbar zu kurz: Es sind nicht die Vieltrinker oder die bei den Risikoindikatoren auffallenden Personen, die Alkohol und Sex kombinieren. Das situationsspezifische Vorgehen erscheint sehr viel präziser, aber erst die Vertiefung durch konkret unterlassene Schutzmassnahmen oder eingegangene Praktiken erlauben die Identifizierung 
von Personen, die tatsächlich "at risk" waren. Alkoholkonsum hängt hier offenbar nicht mit Risikoverhalten zusammen und wo beides zusammentraf, stellten wir keinen erhöhten Anteil von Personen mit Risiko fest.

Die Ende der 80er Jahre entwickelten Fragebögen enthalten durch den Einbezug unterschiedlicher Forschungskonzepte ein grosses Potential. Einige Schwachpunkte wie die mangelnde Präzision bei den allgemeinen Angaben zum Kondomgebrauch wurden schon für die Nachfolgebefragungen verbessert. Ein anderer gravierender Mangel ist dagegen nie behoben worden: Die Anzahl der Partner wird nicht erfragt. Ohne Anleitung zur Auswertung verführen die Instrumente zudem zur Entwicklung von Indikatoren, die dann Vergleiche verschiedener Forschergruppen untereinander erschweren.

Wir bildeten drei gängige Risikoindizes (den dichotomen, den vierstufigen sowie den MACS-Index) nach und gelangten hinsichtlich Alkoholkonsum zu ähnlichen Ergebnissen. Das ist weiter nicht überraschend, basieren sie doch alle auf dem ereignisunspezifischen Ansatz. Eine Empfehlung für den Gebrauch der Indikatoren ist nicht von der Forschungsfragestellung zu trennen und richtet sich nach dem Verwendungszweck. Die Indizes haben alle ihre Berechtigung, wenn bei breit angelegten Untersuchungen Risikoverhalten und Veränderungen dieses Verhaltens im Mittelpunkt stehen. Sie erscheinen hingegen unzweckmässig, wenn es darum geht, sie mit anderen Einflussgrössen, die mit sexuellem Risikoverhalten zusammenhängen, in Beziehung zu setzen. Bei Untersuchungen mit einer HIV-infizierten Studienpopulation erscheint uns die Weiterentwicklung des MACS-Index aufgrund des Infektionsrisikos zweckmässiger, dieser berücksichtigt neben rezeptivem auch insertiven Anal- verkehr ${ }^{44}$. Interessant wäre es zudem, die Sicht der Betroffenen durch die von ihnen vermutete Ursache (z.B. Alkoholkonsum) für unsafes Sexualverhalten zu erfragen und diese mit konkreten Fragen zum Ereignis (z.B. der bei diesem Ereignis eingenommenen Trinkmenge) zu kombinieren. Ausgangsbasis ist dann das Ereignis selber, es könnte dann mit Fragen zum Ereignis vertieft und mit weiteren (auch situationstypischen und ereignisunspezifischen)
Angaben ergänzt werden. Die von uns verwendeten Instrumente leisten dies nur ansatzweise bei Alkohol- und Drogenkonsum (wobei ereignisrelevante Trinkmenge und etwaiger Rauschzustand nicht erfragt wurden), offen bleiben weitere persönliche Aspekte (wie psychisches und körperliches Wohlbefinden) und situationale Komponenten (wie beispielsweise psychischer Druck oder Überredungskünste des Partners), die zum sexuellen Risiko geführt haben.

\section{Summary}

Does alcohol consumption correlate with sexual risk behavior? A conceptional discussion based on a sample of HIV-infected gay men

Does sexual risk behavior and alcohol consumption correlate or is sexual risk behavior due to alcohol consumption? These questions are controversially discussed in the literature. We investigated whether the two different methodological concepts (g/obal association, e.g. not specific to the critial incident and situational association) cause these discrepancies in a sample of 64 HIV-infected gay men. There was no significant global association between alcohol consumption and sexual risk behavior. Using the situational approach, drinking was not related to sexual risk behavior. The combination of these two concepts may help to identify. persons at risk.

\section{Résumé}

Est-ce que la consommation d'alcohol a une corrélation avec le comportement sexual à risque? Une discussion conceptionelle basée sur une population de personnes à comportement homosexuel infectées avec le SIDA

Est-ce que la consommation d'alcohol a une corrélation avec le comportement sexuel à risque óu est-ce que la consommation d'alcohol mène au comportement sexuel à risque? Ce problème est controversement discuté dans la litérature à l'heure actuelle. Cette investigation a pour but de démontrer si les deux concepts (association globale c'est à dire non spécific à un évènement et association situationelle) expliquent les résultats dans une population de 64 personnes à comportement homosexuel infectées avec le SIDA. Ni l'association globale ni l'association situationelle du comportement sexuel avec la consommation d'alcohol démontrent des corrélations significantes. La combinaison des deux concepts pourrait aider à identifier des personnes avec un comportement sexuel à risque. 


\section{Literaturverzeichnis}

1 Williams DI, Stephenson JM, Hart $G J$, et al. A case control study of HIV seroconversion in gay men, 1988-1993: What are the current risk factors? Genitourin Med 1996; 72:193-196.

2 Hickson FCI, Reid DS, Davies PM, et al. No aggregate change in homosexual risk behaviour among gay men attending the Gay Pride festival, United Kingdom, 1993 1995. AIDS 1996; 10:771-774.

3 WHO Regional Office for Europe. Alcohol and HIV/AIDS. Alcohol Plan and Global Programme on AIDS. Report on a Consultation. Report EUR/GPA 233, 1994.

4 Stall RD, McKusick L, Wiley J, et al. Alcohol and drug use during sexual activity and compliance with safe sex guidelines for AIDS. The AIDS behavioral research project. Health Education Quarterly 1986; 13:359-371.

5 Stall RD, Coates TJ, Hoff C. Behavioral risk reduction for HIV infection among gay and bisexual men. Am Psychologist 1988; 43:878-885.

6 Bagnall G, Plant MA, Warwick W. Alcohol, drugs and AIDS-related risks: Results from a prospective study. AIDS-Care 1990;2:309-317.

7 Plant $M$. Alcohol, sex and AIDS. Alcohol and Alcoholism 1990; 25:293-301.

8 Bagnall $G$, Plant MA. HIV/AIDS risks, alcohol and illicit drug use among young adults in areas of high and low rates of HIV infection. AIDS Care 1991; 3:355-361.

9 Shafer MA, Boyer CB. Psychosocial and behavioral factors associated with risk of sexually transmitted diseases, including human immunodeficiency virus infection, among urban high school students. J Pediatrics 1991; 119:826-833.

10 Penkower L, Dew MA, Kingsley L, et al. Behavioral, health and psychosocial factors and risk for HIV infection among sexually active homosexual men: the multicenter AIDS cohort study. Am J Public Health 1991; 81:194-196.
11 Clapper RL, Lipsitt LP. A retrospective study of risk-taking and alcohol-mediated unprotected intercourse. J of Substance Abuse 1991; 3:91-96.

12 McEwan RT, McCallum A, Bhopal $R S$, et al. Sex and the risk of HIV infection: The role of alcohol. $\mathrm{Br} \mathrm{J}$ of Addiction 1992; 87:577-587.

13 Calsyn DA, Saxon AJ, Wells EA, et al. Longitudinal sexual behavior changes in injecting drug users. AIDS 1992; 6:1207-1211.

14 Paul JP, Stall R, Davis F. Sexual risk for HIV transmission among gay/ bisexual men in substance-abuse treatment. AIDS Educ Prev 1993; $5: 11-24$.

15 Lowry R, Holtzman D, Truman BI, et al. Substance use and HIV-related sexual behaviors among US high school students: are they related? Am J Public Health 1994; 84: $1116-1120$.

16 Caetano $R$, Hines $A M$. Alcohol, sexual practices, and risk of AIDS among blacks, Hispanics, and whites. J Acquir Immune Defic Syndr Hum Retrovirol 1995; 10: 554-561.

17 Shillington $A M$, Cottler $L B$, Compton WM III, et al. Is there a relationship between "heavy drinking" and HIV high risk sexual behaviors among general population subjects? It J Addict 1995; 30:1453-1478.

18 Simkins L. Risk of HIV transmission in sexual behaviors of college students. Psychol Rep 1995; 76:787-799.

19 Latkin CA, Mandell W, Vlahov D. The relationship between risk networks' patterns of crack cocaine and alcohol consumption and HIVrelated sexual behaviors among adult injection drug users: a prospective study. Drug Alcohol Dependence 1996; 42:175-181.

20 Leigh BC. Alcohol consumption and sexual activity as reported with a diary technique. $J$ of Abnormal Psychology 1993; 102:490-493.

21 Gold RS, Skinner MJ. Situational factors and thought processes associated with unprotected inter- course in young gay men. AIDS 1992; 6:1021-1030.

22 Temple $M T$, Leigh BC, Schafer J. Unsafe sexual behavior and alcohol use at the event level: results of a national survey. J Acquir Immune Defic Syndr 1993; 6:393-401.

23 Morgan-Thomas $R$, Plant MA, Plant ML. Alcohol, AIDS risks and sex industry clients. Results from a Scottish study. Drug and Alcohol Dependence 1990; 26:265-269.

24 Plant ML, Plant MA, Thomas RM. Alcohol, AIDS risk and commercial sex: Some preliminary results from a Scottish study. Drug and Alcohol Dependence 1990; 25: 51-55.

25 Bolton $R$, Vincke $J$, Mak $R$, et al. Alcohol and risky sex: In search of an elusive connection. Medical Anthropology 1992; 14 : 323-363.

26 Weatherburn $P$, Davies $P M$, Hickson FCL, et al. No connection between alcohol use and unsafe sex among gay and bisexual men. AIDS 1993; 7:115-119.

27 Leigh BC, Miller P. The relationship of substance use with sex to the use of condoms among young adults in two urban areas of Scotland. AIDS Educ Prev 1995; 7:278-284.

28 Läuchli SR. Einfluss von Alkoholkonsum auf das Safer Sex Verhalten. Medizinische Dissertation, Institut für Sozial- und Präventivmedizin der Universität Zürich, 1996.

29 McCusker J, Westenhouse J, Stoddard AM. Use of drugs and alcohol by homosexually active men in relation to sexual practices. J Acquir Immune Defic Syndr 1990; 3:729-736.

30 Traeen $B$, Lewin $B$. Casual sex among Norwegian adolescents. Archives of Sexual Behavior 1992; $21: 253-269$.

31 Leigh BC. Alcohol and unsafe sex: An overview of research and theory. In: Seminara D, Pawlowski A, Watson R, eds. Alcohol, immunomodulation and AIDS, Liss, New York, 1990: 35-46. 
32 Leigh $B C$, Stall $R$. Substance use and risky sexual behavior for exposure to AIDS. Issues in methodology, interpretation and prevention. Am Psychologist 1993; 48:10351045.

33 Stall RD, Leigh BC. Understanding the relationship between drug or alcohol use and high risk sexual activity for HIV transmission: where do we go from here? Addiction 1994; 89:131-134.

34 Donovan C, McEwan R. A review of the literature examining the relationship between alcohol use and HIV-related risk-taking in young people. Addiction 1995; 90:319_ 328.

35 Ledergerber $B$, von Overbeck $J$, Egger $M$, et al. The Swiss HIV Cohort Study: rationale, organisation and selected baseline characteristics. Soz Praeventivmed 1994; 39:387-394.

36 Heusser R, Tschopp A, Beuttler HJ, et al. Determinants of condom use - results from the Swiss HIV cohort Study. Abstract PoD5478, Abstract Book, Vol. 2, VIIIth International Conference on AIDS, Amsterdam, July $19-24$ th 1992 .
37 Robins AG, Dew MA, Davidson $S$, et al. Psychosocial factors associated with risky sexual behavior among HIV-seropositive gay men. AIDS Educ Prev 1994; 6:483492.

38 Sieber $M$, unter Mitwirkung von Fäh $M$, Niklowitz $M$. Die Bedeutung des HIV-Tests für die AidsPrävention. Bern: Stämpfli, 1995.

39 Valdiserri RO, Lyter D, Leviton $L C$, et al. Variables influencing condom use in a cohort of gay and bisexual men. Am J Public Health 1988; 78:801-805.

40 Weatherburn P, Hunt AJ, Davies $P M$, et al. Condom use in a large cohort of homosexually active men in England and Wales. AIDS Care 1991; 3:31-41.

41 Mayes-Dickerson $S$, Elsesser $V$, Hopkins-Schaefer $J$, et al. Sexual practices and AIDS knowledge among women partners of HIVinfected hemophiliacs. Publ Hlth Rep 1992; 107:504-514.

42 Ostrow DG, Beltran E, Joseph J. Sexual behavior research on a cohort of gay men, 1984 - 1990: Can we predict how men will respond to interventions? Arch Sex Behav
$1994 ; 23: 531-552$.

43 Fahrenkrug $H$. Alkoholkonsum und Alkoholmissbrauch. In: Weiss $W$, Hrsg. Gesundheit in der Schweiz, Zürich: Seismo 1993: 235-249.

44 Stall $R$, Ekstrand $M$, Pollack $L$, et al. Relapse from safer sex: The next challenge for AIDS prevention efforts. J Acquir Immune Defic Syndr 1990; $3: 1181-1187$.

\section{Danksagung}

Diese Untersuchung wurde unterstützt durch den Schweizerischen Nationalfonds (Nr. 4026-26868) und durch die EMDO-Stiftung.

\section{Korrespondenzadresse}

Dr. Dominique Eich-Höchli

Departement Innere Medizin

Abteiluntg für Infektionskrankheiten und Spitalhygiene

Universitätsspital Zürich

Rämistrasse 100

CH-8091 Zürich

\section{Anhang}

\section{Alkoholkonsum}

\section{Trinkhäufigkeit}

Wie häufig trinken Sie alkoholische Getränke?

(Antwortvorgaben: nie - seltener $1 \mathrm{mal} /$ Monat - 2-3mal/Monat 1-2mal/Woche - 3-4mal/Woche täglich)

\section{Trinkmenge}

Wenn Sie Alkohol trinken, wieviel trinken Sie dann pro Gelegenheit? Geben Sie die Menge in Anzahl Gläser an.

(Antwortvorgaben: 1 oder 2 Gläser - 3 oder 4 Gläser - 5 oder 6 Gläser

-7 oder mehr Gläser)

\section{Rausch}

In den letzien 6 Monaten, wie oft hatten Sie einen "Rausch"? (d.h. dass Sie die berauschende Wirkung des Alkohols deutlich gespürt haben)

(Antwortvorgaben: nie - 1mal 2-4mal - 5-9mal - 10-19mal 20-50mal - über 50mal)

\section{MACS-Alkohol-Trinkfrequenz}

Die Alkohol-Trinkfrequenz der MACS errechnet sich aus der Anzahl Drinks (= Gläser mit alkoholischem Getränk) pro Monat, also aus dem Produkt von Trinkhäufigkeit und Trinkmenge. Dieses Rohmass wird trichotomisiert ${ }^{42}$ : niedriger Konsum (bis 12 Drinks pro Monat), mittlerer Konsum (13 bis 59 Drinks) und hoher Konsum (über 59 Drinks)

\section{Sexuelles Schutz- und Risikoverhalten}

Sexuelle Aktivität

Allgemein

Hatten Sie in den letzten 6 Monaten homosexuelle Kontakte?

Anzahl und Art der Kontakte

Aktiv (gleich aufgebaut: passiv) 


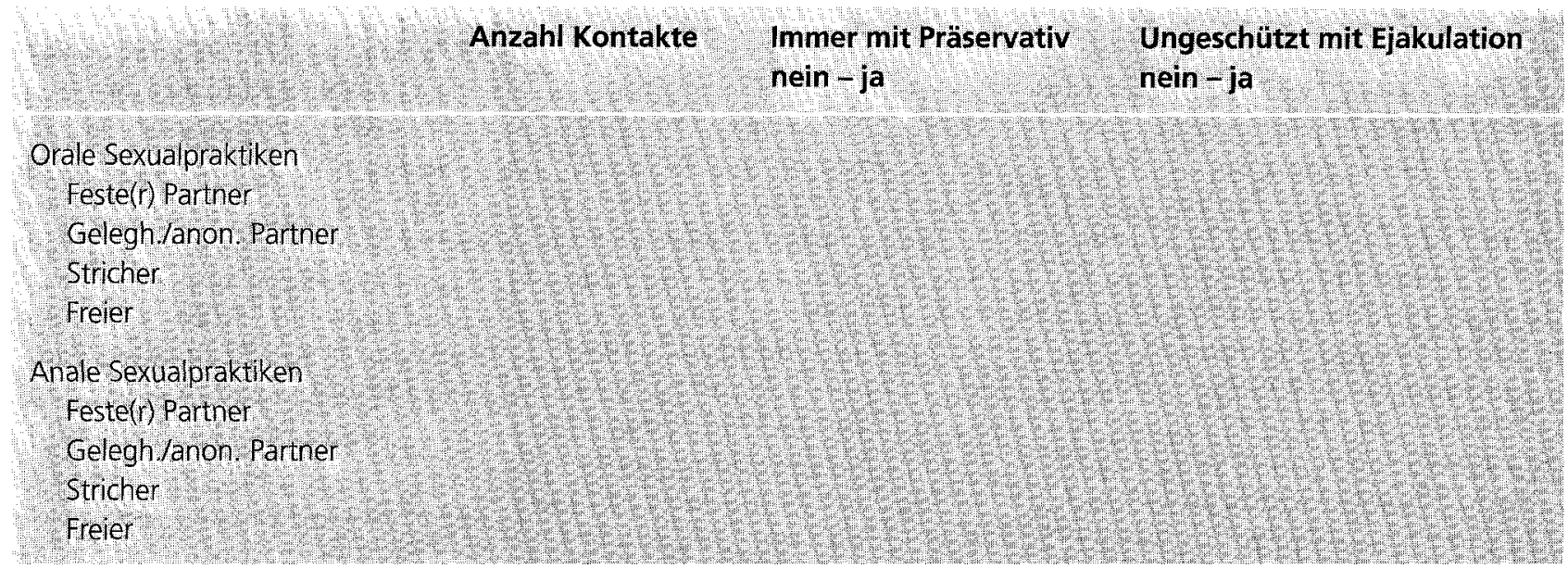

Sexuelle Risikoindikatoren

\section{Dichotomer Indikator}

Als Sexualpraktiken mit substantieller Infektionswahrscheinlichkeit gelten aktiver oder passiver Analverkehr. Der Indikator weist zwei Stufen auf:

1. Es wurden immer Kondome gebraucht bzw.

2. der Gebrauch ist mindestens einmal unterlassen worden.

Dieser Indikator wird getrennt für Kontakte mit festen und Gelegenheitspartnern berechnet. Kontakte mit Strichern oder Freiern wurden von der Studienpopulation nicht genannt.

\section{Vierstufiger Indikator ${ }^{39-41}$}

Wie beim dichotomen Indikator gelten aktiver oder passiver Analverkehr als Sexualpraktiken mit substantieller Infektionswahrscheinlichkeit.

Dieser Indikator weist vier Stufen auf:

1. Keine analen Kontakte

2. Sämtliche analen Kontakte geschützt;
3. Teils geschützte, teils ungeschützte anale Kontakte;

4. Immer ungeschützte anale Kontakte.

Dieser Indikator wird für Kontakte mit festen und Gelegenheitspartnern berechnet. Kontakte mit Strichern oder Freiern wurden von der Studienpopulation nicht genannt.

\section{MACS-Risikoindikator ${ }^{42}$}

Dieser Indikator ist vierstufig.

1. Kein Risiko: Keine sexuellen Kontakte oder Partner.

2. Niedriges Risiko: Sexuelle Kontakte mit Partner(n), aber entweder

kein rezeptiver Analverkehr oder

rezeptiver Analverkehr nur mit festem Partner und konsistentem Kondomgebrauch.

3. Erhöhtes Risiko: Entweder konsistenter Kondomgebrauch bei rezeptivem Analverkehr mit mehreren Partnern oder ungeschützter rezeptiver Analverkehr, beschränkt auf den festen Partner.

4. Hohes Risiko: ungeschützter rezeptiver Analverkehr, mehrere Partner.

\section{Alkoholkonsum und sexuelle Kontakte}

\section{Eingangsfrage}

Hatten Sie während der letzten 6 Monate sexuelle Kontakte unter Alkoholeinfluss? (d.h. in durch Alkohol berauschtem Zustand)

(Antwortvorgaben: nein-ja)

\section{Anzahl Kontakte unter Alkohol-} einfluss und Partnertypen

Wenn ja:

Falls Sie in den letzten 6 Monaten sexuelle Kontakte unter Alkoholeinfluss hatten, versuchen Sie sich $z u$ erinnern, mit wem und wie oft dies geschah?

Im folgenden unterscheiden wir vier Partnertypen. a) feste b) Gelegenheits- c) anonyme Partner und $d$ ) Prostituierte. (...)

Feste Partner: (Beziehung mit über 3 Monaten Dauer, schliesst gleichzeitige Beziehungen zu anderen Partnern nicht aus).

Wie oft waren Sie in den letzten 6 Monaten von Alkohol berauscht, als Sie sexuelle Kontakte zu Ihrem(n) festen Partner(n) hatten. 


$\begin{array}{lll}\text { Nie } & \begin{array}{l}\text { Selten } \\ \text { (unter }\end{array} & \begin{array}{l}\text { Gelegentlich } \\ (20-50 \% \ldots\end{array} \\ 10 \% * \ldots) & \text { der Kontakte) }\end{array}$

Weibliche Partner

Männliche Partner

* d. h. in weniger als $10 \%$ aller sexuellen Kontakte mit festen Partnern stand ich unter Alkoholeinfluss.
Häufig

(über $\mathbf{5 0} \% \ldots$. . )
Immer

\section{der Kontakte)}

Ebenso für die anderen Partnertypen (Kontakte mit Strichern/Freiern wurden keine genannt):

Gelegenheitspartner: (Gelegentliche sexuelle Beziehungen mit Personen aus dem Bekanntenkreis). Anonyme Partner: (Partner, die man nachher nicht mehr erreichen kann).

Prostituierte(r).

Sexualpraktiken

Mundverkehr

Scheidenverkehr

Analverkehr:
Folgen des Alkoholkonsums bezüglich Sexualpraktiken und Schutzmassnahmen

\section{Eingangsfrage}

Haben Sie in den letzten 6 Monaten unter Alkoholeinfluss Vorsichtsmassnahmen bezüglich „Safer Sex" unterlassen, die Sie nüchtern einhalten würden?

(Antwortvorgaben: nein - ja)
Aktiv

mit

Präservativ ohne

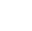

\section{Sexualpraktiken}

Ich bin Sexualpraktiken eingegangen, die ich nüchtern vermieden hätte.

(Antwortvorgaben: nein - ja) Falls ja, welche?

\section{Schutzmassnahmen}

Ich habe bei unten stehenden Praktiken kein Präservativ verwendet.

\section{Sexualpraktiken}

Mundverkethr

Scheidenverkehr

Analverkehr
Aktiv

Passiv 